\title{
A new Easton theorem for supercompactness and level by level equivalence \\ by
}

\author{
Arthur W. APTER \\ Presented by Czestaw BESSAGA
}

\begin{abstract}
Summary. We establish a new Easton theorem for the least supercompact cardinal $\kappa$ that is consistent with the level by level equivalence between strong compactness and supercompactness. This theorem is true in any model of ZFC containing at least one supercompact cardinal, regardless if level by level equivalence holds. Unlike previous Easton theorems for supercompactness, there are no limits on the Easton functions $F$ used, other than the usual constraints given by Easton's theorem and the fact that if $\delta<\kappa$ is regular, then $F(\delta)<\kappa$. In both our ground model and the model witnessing the conclusions of our theorem, there are no restrictions on the structure of the class of supercompact cardinals.
\end{abstract}

1. Introduction and preliminaries. Say that a model of ZFC witnesses level by level equivalence between strong compactness and supercompactness (which we will henceforth abbreviate as just level by level equivalence) iff for every measurable cardinal $\kappa$ and every regular cardinal $\lambda>\kappa$, $\kappa$ is $\lambda$ strongly compact iff $\kappa$ is $\lambda$ supercompact, except possibly if $\kappa$ is a measurable limit of cardinals $\delta$ which are $\lambda$ supercompact. Models containing supercompact cardinals which also witness the level by level equivalence between strong compactness and supercompactness and satisfy GCH were first constructed in [5].

We remark that the exceptions in the previous paragraph are provided by a theorem of Menas [12, who showed that if $\kappa$ is a measurable limit of cardinals $\delta$ which are $\lambda$ strongly compact, then $\kappa$ is $\lambda$ strongly compact but

2010 Mathematics Subject Classification: 03E35, 03E55.

Key words and phrases: supercompact cardinal, strongly compact cardinal, strong cardinal, level by level equivalence between strong compactness and supercompactness, Easton theorem.

Received 30 July 2016.

Published online 17 July 2017. 
need not be $\lambda$ supercompact. When this situation occurs, we will henceforth say that $\kappa$ is a witness to the Menas exception at $\lambda$. If $\kappa$ is measurable and for every regular cardinal $\lambda>\kappa, \kappa$ is $\lambda$ strongly compact iff $\kappa$ is $\lambda$ supercompact, then we will say that $\kappa$ is a witness to level by level equivalence between strong compactness and supercompactness.

We continue now with the main narrative. In [1] and [2], the following theorems were proven.

ThEOREM $1([1])$. Let $V \vDash " Z F C+G C H+$ Level by level equivalence between strong compactness and supercompactness holds $+\mathcal{K} \neq \emptyset$ is the class of supercompact cardinals $+\kappa$ is the least supercompact cardinal". Let $A=\{\delta \leq \kappa \mid \delta$ is either a strong cardinal or the regular limit of strong cardinals\}. Suppose that $F: A \rightarrow \kappa, F \in V$ is a function with the following properties.

(1) $F(\delta) \in\left(\delta, \delta^{*}\right)$ is a cardinal, where $\delta^{*}$ is the least strong cardinal above $\delta$.

(2) $\operatorname{cof}(F(\delta))>\delta$.

(3) If $\delta \in A$ is $\lambda$ supercompact for $\lambda>\delta$, then there is an elementary embedding $j: V \rightarrow M$ witnessing the $\lambda$ supercompactness of $\delta$ generated by a supercompact ultrafilter over $P_{\delta}(\lambda)$ such that either $j(F)(\delta)=F(\delta)=\delta^{+}$ or $j(F)(\delta)=F(\delta)=\delta^{++}$.

There is then a cardinal and cofinality preserving partial ordering $\mathbb{P} \in V$ such that $V^{\mathbb{P}} \vDash " Z F C+\mathcal{K}$ is the class of supercompact cardinals (so $\kappa$ is the least supercompact cardinal) + Level by level equivalence between strong compactness and supercompactness holds + For every $\delta \in A, 2^{\delta}=F(\delta)$ ".

Theorem $2([2])$. Let $V \vDash " Z F C+G C H+$ Level by level equivalence between strong compactness and supercompactness holds $+\mathcal{K} \neq \emptyset$ is the class of supercompact cardinals $+\kappa$ is the least supercompact cardinal". Let $A=\{\delta \leq \kappa \mid \delta$ is a regular cardinal which is not the successor of a singular cardinal and $\neg \exists \gamma<\delta[\gamma$ is $\alpha$ supercompact for every $\alpha<\delta]\}$. Suppose that $F: A \rightarrow \kappa, F \in V$ is a function with the following properties.

(1) If $\delta_{1}<\delta_{2}$, then $F\left(\delta_{1}\right) \leq F\left(\delta_{2}\right)$.

(2) $F(\delta) \in\left(\delta, \delta^{\prime}\right)$ is a cardinal, where $\delta^{\prime}$ is the least Mahlo cardinal above $\delta$.

(3) $\operatorname{cof}(F(\delta))>\delta$.

(4) If $\delta \in A$ is $\lambda$ supercompact for $\lambda>\delta$, then there is an elementary embedding $j: V \rightarrow M$ witnessing the $\lambda$ supercompactness of $\delta$ generated by a supercompact ultrafilter over $P_{\delta}(\lambda)$ such that either $j(F)(\delta)=F(\delta)=\delta^{+}$ or $j(F)(\delta)=F(\delta)=\delta^{++}$.

There is then a cardinal and cofinality preserving partial ordering $\mathbb{P} \in V$ such that $V^{\mathbb{P}} \vDash " Z F C+\mathcal{K}$ is the class of supercompact cardinals (so $\kappa$ is the least supercompact cardinal) + Level by level equivalence between strong compactness and supercompactness holds + For every $\delta \in A, 2^{\delta}=F(\delta)$ ". 
TheOREm 3 ([2]). Let $V \vDash " Z F C+G C H+$ Level by level equivalence between strong compactness and supercompactness holds $+\mathcal{K} \neq \emptyset$ is the class of supercompact cardinals $+\kappa$ is the least supercompact cardinal". Let $A=$ $\{\delta \leq \kappa \mid \delta$ is either a strong cardinal or the regular limit of strong cardinals $\}$. Suppose that $F: A \rightarrow \kappa, F \in V$ is a function with the following properties.

(1) $F(\delta) \in\left(\delta, \delta^{*}\right)$ is a cardinal, where $\delta^{*}$ is the least strong cardinal above $\delta$.

(2) $\operatorname{cof}(F(\delta))>\delta$.

(3) If $\delta \in A$ is $\lambda$ supercompact for $\lambda \geq \delta^{+16}$, then there is an elementary embedding $j: V \rightarrow M$ witnessing the $\lambda$ supercompactness of $\delta$ generated by a supercompact ultrafilter over $P_{\delta}(\lambda)$ such that $j(F)(\delta)=F(\delta)=\delta^{+17}$.

There is then a cardinal and cofinality preserving partial ordering $\mathbb{P} \in V$ such that $V^{\mathbb{P}} \vDash " Z F C+\mathcal{K}$ is the class of supercompact cardinals (so $\kappa$ is the least supercompact cardinal) + Level by level equivalence between strong compactness and supercompactness holds + For every $\delta \in A, 2^{\delta}=F(\delta)$ ".

As in 2] (from which we quote), the use of $\delta^{+16}$ and $\delta^{+17}$ in the statement of Theorem 3 is for ease of presentation and comprehensibility. In essence, $\delta^{+17}$ should be seen as representing any suitable regular cardinal $\lambda$ for which it will be the case that $2^{\delta}=\lambda$. The cardinals $\delta^{+16}$ and $\delta^{+17}$ may be viewed as a form of "wild card" standing in for the more general possibilities. Readers are referred to [2] for additional details.

In Theorems 173, there are severe restrictions placed on the witnessing Easton functions $F$. In particular, $F(\delta)$ has harsh constraints placed on the values it may take, and specifically must be well-behaved with respect to the appropriate supercompactness embeddings. This raises the general question of whether it is possible to prove an Easton theorem for the least supercompact cardinal consistent with level by level equivalence in which the only limitation placed on the witnessing Easton function $F$ (beyond the usual ones for Easton functions) is that for $\delta$ below the least supercompact cardinal $\kappa, F(\delta)<\kappa$.

The purpose of this paper is to answer this question in the affirmative. More specifically, we will prove the following theorem.

TheOREM 4. Let $V \vDash " Z F C+G C H+$ Level by level equivalence between strong compactness and supercompactness holds $+\mathcal{K} \neq \emptyset$ is the class of supercompact cardinals $+\kappa$ is the least supercompact cardinal". Let $A=\{\delta<\kappa \mid$ $\delta$ is a nonmeasurable regular cardinal and $\neg \exists \gamma<\delta[\gamma$ is $\alpha$ supercompact for every $\alpha<\delta]\}$. Then $A$ is a stationary subset of $\kappa$. Further, suppose that $F: A \rightarrow \kappa, F \in V$ is a function with the following properties.

(1) If $\delta_{1}<\delta_{2}$, then $F\left(\delta_{1}\right) \leq F\left(\delta_{2}\right)$.

(2) $\operatorname{cof}(F(\delta))>\delta$.

(3) $F(\delta)<\kappa$ is a cardinal. 
There is then a cardinal and cofinality preserving partial ordering $\mathbb{P} \in V$ which also preserves the stationarity of $A$ such that $V^{\mathbb{P}} \vDash " Z F C+\mathcal{K}$ is the class of supercompact cardinals (so $\kappa$ is the least supercompact cardinal) + Level by level equivalence between strong compactness and supercompactness holds + For every $\delta \in A, 2^{\delta}=F(\delta)$ ".

Restrictions (1) and (2) are the usual ones for Easton functions. Restriction (3) is necessary since otherwise, for some $\delta<\kappa, V^{\mathbb{P}} \vDash " 2^{\delta} \geq \kappa$ ", contradicting the supercompactness of $\kappa$ in $V^{\mathbb{P}}$.

Thus, it is possible to find a stationary subset $A$ of the least supercompact cardinal $\kappa$ such that an arbitrary Easton function $F$ can be realized on $A$ while preserving all ground model supercompact cardinals, the stationarity of $A$, and level by level equivalence. In addition, the generic extension realizing $F$ will contain the same supercompact cardinals as the ground model. Further, since $F$ is completely arbitrary, it does not have to satisfy any definability constraints, including those given by Menas in [11. Consequently, as our proof (which is quite different from the proofs of Theorems 1-3 and [11, Theorem, Section 18, pp. 83-88]) will show, we have in fact established a completely new Easton theorem for supercompactness, which is valid in any model of $Z F C$ containing at least one supercompact cardinal (including those in which level by level equivalence does not hold).

Before presenting the proof of our theorem, we briefly state some preliminary information. Our notation and terminology will follow that given in [1] and [2]. We do wish to mention a few things explicitly, however. For $\kappa$ a regular cardinal and $\alpha$ an ordinal, $\operatorname{Add}(\kappa, \alpha)$ is the standard Cohen partial ordering for adding $\alpha$ Cohen subsets of $\kappa$. A partial ordering $\mathbb{P}$ is $\kappa$-directed closed for $\kappa$ a regular cardinal if for every directed set of conditions $D$ of size less than $\kappa$, there is a single condition extending each member of $D$. If $G$ is $V$-generic over $\mathbb{P}$, we will abuse notation slightly and use both $V[G]$ and $V^{\mathbb{P}}$ to indicate the universe obtained by forcing with $\mathbb{P}$. We will, from time to time, confuse terms with the sets they denote and write $x$ when we actually mean $\dot{x}$ or $\check{x}$.

We mention that we are assuming familiarity with the large cardinal notions of measurability, strongness, strong compactness, and supercompactness. Interested readers may consult [8].

A corollary of Hamkins' work on gap forcing found in [6, 7] will be employed in the proofs of our theorems. We therefore state as a separate theorem what is relevant for this paper, along with some associated terminology, quoting from [6, 7] when appropriate. Suppose $\mathbb{P}$ is a partial ordering which can be written as $\mathbb{Q} * \dot{\mathbb{R}}$, where $|\mathbb{Q}|<\delta, \mathbb{Q}$ is nontrivial, and $\vdash_{\mathbb{Q}}$ " $\mathbb{R}$ is $\delta^{+}$-directed closed". In Hamkins' terminology of [6, 7], $\mathbb{P}$ admits a gap at $\delta$. In Hamkins' terminology of [6, 7], $\mathbb{P}$ is mild with respect to a cardinal $\kappa$ iff 
every set of ordinals $x$ in $V^{\mathbb{P}}$ of size less than $\kappa$ has a "nice" name $\tau$ in $V$ of size less than $\kappa$, i.e., there is a set $y$ in $V,|y|<\kappa$, such that $\Vdash_{\mathbb{P}}$ " $\tau \subseteq \check{y}$ ". Also, as in the terminology of [6, 7] and elsewhere, an embedding $j: \bar{V} \rightarrow \bar{M}$ is amenable to $\bar{V}$ when $j\lceil A \in \bar{V}$ for any $A \in \bar{V}$. The specific corollary of Hamkins' work from [6, 7] we will be using is then the following.

THEOREM 5 (Hamkins). Suppose that $V[G]$ is a generic extension obtained by forcing with $\mathbb{P}$ that admits a gap at some regular $\delta<\kappa$. Suppose further that $j: V[G] \rightarrow M[j(G)]$ is an embedding with critical point $\kappa$ for which $M[j(G)] \subseteq V[G]$ and $M[j(G)]^{\delta} \subseteq M[j(G)]$ in $V[G]$. Then $M \subseteq V$; indeed, $M=V \cap M[j(G)]$. If the full embedding $j$ is amenable to $V[G]$, then the restricted embedding $j \backslash V: V \rightarrow M$ is amenable to $V$. If $j$ is definable from parameters (such as a measure or extender) in $V[G]$, then the restricted embedding $j\lceil V$ is definable from the names of those parameters in $V$. Finally, if $\mathbb{P}$ is mild with respect to $\kappa$ and $\kappa$ is $\lambda$ strongly compact in $V[G]$ for any $\lambda \geq \kappa$, then $\kappa$ is $\lambda$ strongly compact in $V$.

Theorem 5 implies that if $\kappa$ is supercompact in $V^{\mathbb{P}}$ and $\mathbb{P}$ admits a gap below $\kappa$, then $\kappa$ was supercompact in $V$ as well. In addition, if $\kappa$ is strongly compact in $V^{\mathbb{P}}$ and $\mathbb{P}$ is both mild with respect to $\kappa$ and admits a gap below $\kappa$, then $\kappa$ was also strongly compact in $V$.

2. The proof of Theorem 4. We turn now to the proof of Theorem 4 Suppose $V \vDash$ "ZFC + GCH + Level by level equivalence between strong compactness and supercompactness holds $+\mathcal{K} \neq \emptyset$ is the class of supercompact cardinals $+\kappa$ is the least supercompact cardinal". Let $A$ and $F: A \rightarrow \kappa$ be as in the hypotheses of Theorem 4. We begin by showing that $A$ is a stationary subset of $\kappa$.

LEMMA 2.1. In $V, A=\{\delta<\kappa \mid \delta$ is a nonmeasurable regular cardinal and $\neg \exists \gamma<\delta[\gamma$ is $\alpha$ supercompact for every $\alpha<\delta]\}$ is a stationary subset of $\kappa$.

Proof. Let $j: V \rightarrow M$ be an elementary embedding generated by a normal measure $\mu$ over $\kappa$ such that $M \vDash$ " $\kappa$ is not measurable". By [4, Lemma 2.1] and the succeeding remarks, in both $V$ and $M, \kappa$ is a limit of strong cardinals. Further, for no $\gamma<\kappa$ is it the case that $M \vDash " \gamma$ is $\alpha$ supercompact for every $\alpha<\kappa$ ". This is since otherwise, for $\eta<\kappa$ such that $M \vDash$ " $\eta$ is a strong cardinal", $M \vDash$ " $\gamma$ is $\alpha$ supercompact for every $\alpha<\eta$ ". Thus, by [3, Lemma 1.1], $M \vDash$ " $\gamma$ is supercompact". However, since $M \vDash " j(\gamma)=\gamma$ is supercompact", $V \vDash$ " $\gamma$ is supercompact" as well. This contradicts that $\gamma<\kappa$ and $V \vDash " \kappa$ is the least supercompact cardinal". As $M \vDash$ " $\kappa$ is regular", it is therefore the case that $\kappa \in j(A)$. Hence, $A \in \mu$, from which it immediately follows that $V \vDash$ " $A$ is a stationary subset of $\kappa$ ". This completes the proof of Lemma 2.1. 
We next turn to the definition of the partial ordering $\mathbb{P}$ used in the proof of Theorem 4. We take as our convention for the duration of this paper that all product partial orderings have Easton support. $\mathbb{P}$ will be defined as $\operatorname{Add}(\omega, 1) *\left(\dot{\mathbb{P}}^{1} \times \dot{\mathbb{P}}^{0}\right)$, where both $\mathbb{P}^{1}$ and $\mathbb{P}^{0}$ are products. To define $\mathbb{P}^{1}$ and $\mathbb{P}^{0}$, let $\delta_{0}$ be the least inaccessible cardinal (which is the same in either $V$ or $\left.V_{0}=V^{\operatorname{Add}(\omega, 1)}\right)$. Write $A=A_{0} \cup A_{1}$, where $A_{0}=\left\{\gamma \in A \mid \gamma<\delta_{0}\right\}$ and $A_{1}=\left\{\gamma \in A \mid \gamma \geq \delta_{0}\right\}$. Note that by the definition of $A, A_{0}$ consists of all of the regular cardinals less than $\delta_{0}$. We can now, working in $V_{0}$, let $\mathbb{P}^{1}=$ $\prod_{\gamma \in A_{1}} \operatorname{Add}(\gamma, F(\gamma))$ and $\mathbb{P}^{0}=\prod_{\gamma \in A_{0}} \operatorname{Add}(\gamma, F(\gamma))$. Since by the definitions of $\mathbb{P}^{1}$ and $\mathbb{P}^{0}, \mathbb{P}^{1} \times \mathbb{P}^{0}$ is the Easton support product $\prod_{\gamma \in A} \operatorname{Add}(\gamma, F(\gamma))$, the standard arguments concerning Cohen, iterated, and Easton forcing (see [8]) show that forcing with $\mathbb{P}$ preserves cardinals and cofinalities and $V^{\mathbb{P}} \vDash$ "For every $\gamma \in A, 2^{\gamma}=F(\gamma)$ ".

A few words are perhaps now in order concerning the intuition behind the above definition of $\mathbb{P} . \mathbb{P}$ begins by forcing with $\operatorname{Add}(\omega, 1)$ to introduce a sufficiently low gap, so that Theorem 5 can be applied. The partial orderings $\mathbb{P}^{0}$ and $\mathbb{P}^{1}$ are used in the proofs of Lemmas 2.22 .4 , which is why $\prod_{\gamma \in A} \operatorname{Add}(\gamma, F(\gamma))$ is factored as $\mathbb{P}^{1} \times \mathbb{P}^{0}$.

Lemma 2.2. Suppose $V \vDash " \delta<\lambda$ are such that $\delta$ is $\lambda$ supercompact and $\lambda$ is regular". If $F(\gamma)<\delta$ for every $\gamma<\delta$, then $V^{\mathbb{P}} \vDash$ " $\delta$ is $\lambda$ supercompact".

Proof. Since $|\operatorname{Add}(\omega, 1)|=\omega<\delta$, by the Lévy-Solovay results [10], $V_{0} \vDash$ " $\delta$ is $\lambda$ supercompact". We continue to work in $V_{0}$ for the remainder of the proof of this lemma. By hypothesis, $F(\gamma)<\delta$ for every $\gamma<\delta$. Since $\delta_{0}$ is the least inaccessible cardinal in either $V$ or $V_{0}$, we have $\delta_{0}<\delta$. Hence, $F(\gamma)<\delta$ for every $\gamma \in A_{0}$. Therefore, by the definition of $\mathbb{P},\left|\mathbb{P}^{0}\right|<\delta$. This means by the results of [10] that $V^{\mathbb{P}}=V_{0}^{\mathbb{P}^{1} \times \mathbb{P}^{0}} \vDash$ " $\delta$ is $\lambda$ supercompact" iff $V_{0}^{\mathbb{P}^{1}} \vDash$ " $\delta$ is $\lambda$ supercompact". We thus show that $V_{0}^{\mathbb{P}^{1}} \vDash$ " $\delta$ is $\lambda$ supercompact".

To do this, let $A_{1}=A_{2} \cup A_{3}$, where $A_{2}=\left\{\gamma \in A_{1} \mid \gamma<\delta\right\}$ and $A_{3}=$ $\left\{\gamma \in A_{1} \mid \gamma \geq \delta\right\}$. Write $\mathbb{P}^{1}=\mathbb{Q}^{0} \times \mathbb{Q}^{1}$, where $\mathbb{Q}^{1}=\prod_{\gamma \in A_{3}} \operatorname{Add}(\gamma, F(\gamma))$ and $\mathbb{Q}^{0}=\prod_{\gamma \in A_{2}} \operatorname{Add}(\gamma, F(\gamma))$. By the definitions of $A$ and $\mathbb{P}$ and our hypothesis that $F(\gamma)<\delta$ for $\gamma<\delta$, we infer that $\left|\mathbb{Q}^{0}\right| \leq \delta, \mathbb{Q}^{0}$ is $\delta$-c.c., and the first regular cardinal on which $\mathbb{Q}^{1}$ acts is at least $\lambda^{++}$. Thus, by its definition, $\mathbb{Q}^{1}$ is $\lambda^{++}$-directed closed, so forcing with $\mathbb{Q}^{1}$ over $V_{0}$ adds no new subsets of $\lambda^{+}$. Also, since $V_{0} \vDash \mathrm{GCH}$, we see that $V_{0}^{\mathbb{Q}^{0}} \vDash " 2^{\gamma}=\gamma^{+}$for all cardinals $\gamma \geq \delta$ ", from which it immediately follows that $\left.V_{0}^{\mathbb{Q}^{0}} \vDash " 2^{[\lambda]}\right]^{<\delta}=2^{\lambda}=\lambda^{+"}$. By [8, Lemma 15.19], we hence infer that forcing with $\mathbb{Q}^{1}$ over $V_{0}^{\mathbb{Q}^{0}}$ adds no new subsets of $2^{[\lambda]^{<\delta}}$. This means that $V_{0}^{\mathbb{P}^{1}}=V_{0}^{\mathbb{Q}^{1} \times \mathbb{Q}^{0}}=V_{0}^{\mathbb{Q}^{0} \times \mathbb{Q}^{1}} \vDash " \delta$ is $\lambda$ supercompact" iff $V_{0}^{\mathbb{Q}^{0}} \vDash$ " $\delta$ is $\lambda$ supercompact". We consequently now show that $V_{0}^{\mathbb{Q}^{0}} \vDash$ " $\delta$ is $\lambda$ supercompact" by considering the following two cases. 
CAsE 1: $\left|\mathbb{Q}^{0}\right|<\delta$. Once again, by the results of $[10], V_{0}^{\mathbb{Q}^{0}} \vDash " \delta$ is $\lambda$ supercompact".

CASE 2: $\left|\mathbb{Q}^{0}\right|=\delta$. Let $j: V_{0} \rightarrow M$ be an elementary embedding witnessing the $\lambda$ supercompactness of $\delta$ generated by a supercompact ultrafilter over $\delta$. By GCH in both $V_{0}$ and $M, M \vDash " ~ \delta$ is $\alpha$ supercompact for every $\alpha<\lambda$ ". The definition of $\mathbb{P}$ therefore implies that $j\left(\mathbb{Q}^{0}\right)=\mathbb{Q}^{0} \times \mathbb{R}$, where the first cardinal in $M$ on which $\mathbb{R}$ acts is at least $\lambda^{+}$. Further, the definition of $\mathbb{P}$ implies that $M \vDash "|\mathbb{R}| \leq j(\delta)$ and $\mathbb{R}$ is $\lambda^{+}$-directed closed". Consequently, because $M^{\lambda} \subseteq M, V_{0} \vDash$ " $\mathbb{R}$ is $\lambda^{+}$-directed closed" as well. In addition, the number of dense open subsets of $\mathbb{R}$ present in $M$ is at most $2^{j(\delta)}$, which by $\mathrm{GCH}$ in both $V_{0}$ and $M$ is $(j(\delta))^{+}=j\left(\delta^{+}\right)$. However, since $\lambda \geq \delta^{+}$, by GCH in $V_{0}$ it follows that

$$
\begin{aligned}
\left|j\left(\delta^{+}\right)\right| & =\mid\left\{f \mid f: P_{\delta}(\lambda) \rightarrow \delta^{+} \text {is a function }\right\} \mid \\
& =\mid\left\{f \mid f: \lambda \rightarrow \delta^{+} \text {is a function }\right\} \mid \\
& =\mid\{f \mid f: \lambda \rightarrow \lambda \text { is a function }\} \mid=2^{\lambda}=\lambda^{+} .
\end{aligned}
$$

We can therefore use the usual diagonalization arguments (as given, e.g., in the construction of the generic object $G_{1}$ in [4, Lemma 2.4]) to build in $V_{0}$ an $M$-generic object $H$ over $\mathbb{R}$. More specifically, let $\left\langle D_{\alpha} \mid \alpha<\lambda^{+}\right\rangle$enumerate in $V_{0}$ the dense open subsets of $\mathbb{R}$ present in $M$. As $M^{\lambda} \subseteq M$, by the $\lambda^{+}$-directed closure of $\mathbb{R}$ in $V_{0}$, we may work in $V_{0}$ and form an increasing sequence $\left\langle q_{\alpha} \mid \alpha<\lambda^{+}\right\rangle$of elements of $\mathbb{R}$ such that $q_{\alpha} \in D_{\alpha}$ for every $\alpha<\lambda^{+}$. Then $H=\left\{p \in \mathbb{R} \mid \exists \alpha<\lambda^{+}\left[q_{\alpha} \geq p\right]\right\}$ is our desired generic object. Because $H \in V_{0}$ and $M \subseteq V_{0}, M[H] \subseteq V_{0}$. Hence, if $G$ is $V_{0}$-generic over $\mathbb{Q}^{0}$, $G$ is also $M[H]$-generic over $\mathbb{Q}^{0}$. Since $j^{\prime \prime} G \subseteq G \times H$ and by the product lemma, $M[H][G]=M[G][H], j$ lifts in $V_{0}[G]$ to $j: V_{0}[G] \rightarrow M[G][H]$. Thus, $V_{0}^{\mathbb{Q}^{0}} \vDash " \delta$ is $\lambda$ supercompact".

Cases 1 and 2 complete the proof of Lemma 2.2.

LEMMA 2.3. $V^{\mathbb{P}} \vDash$ "Every measurable cardinal $\delta$ is either a witness to level by level equivalence or is a witness to the Menas exception at $\lambda$ for some $\lambda>\delta$ ", i.e., $V^{\mathbb{P}} \vDash$ "Level by level equivalence holds".

Proof. Suppose $V^{\mathbb{P}} \vDash " \delta<\lambda$ are such that $\delta$ is $\lambda$ strongly compact and $\lambda$ is regular". We prove Lemma 2.3 by considering the following three cases, where we adopt throughout the notation and terminology of Lemma 2.2 .

CASE 1: $\delta>\kappa$. When this occurs, then by its definition, $|\mathbb{P}|=\kappa<\delta$. Consequently, since $V \vDash$ "Level by level equivalence holds", by the results of [10], $V^{\mathbb{P}} \vDash$ "Either level by level equivalence holds at $\delta$, or $\delta$ is a witness to the Menas exception at $\lambda$ ". 
CASE 2: $\delta=\kappa$. Then the analysis given in Lemma 2.2 (specifically, Case 2) shows that for any regular $\lambda>\kappa, V^{\mathbb{P}} \vDash$ " $\kappa$ is $\lambda$ supercompact". Thus, $V^{\mathbb{P}} \vDash " \kappa$ is supercompact". In this situation, $A_{3}=\emptyset$, and $\mathbb{Q}^{1}$ is trivial forcing.

CASE 3: $\delta<\kappa$. It is then true that $V^{\mathbb{P}}=V^{\operatorname{Add}(\omega, 1) *\left(\dot{\mathbb{P}}^{1} \times \dot{\mathbb{P}}^{0}\right)} \vDash " \delta$ is $\lambda$ strongly compact". Note it must be true that for all $\gamma \in A_{0}, F(\gamma)<\delta$. This is since if $\gamma \in A_{0}$, then $\gamma<\delta_{0}<\delta$, so if $F(\gamma) \geq \delta$, then by the definition of $\mathbb{P}$, $V^{\mathbb{P}} \vDash " 2^{\gamma} \geq \delta$ ". This contradicts the fact that $V^{\mathbb{P}} \vDash$ " $\delta$ is $\lambda$ strongly compact". Because $V^{\mathbb{P}} \vDash$ " $\delta$ is Mahlo" and forcing cannot create a new Mahlo cardinal, $V \vDash$ " $\delta$ is Mahlo and hence inaccessible". Since $\delta_{0}<\delta$ and $\delta$ is inaccessible, it therefore follows by the definition of $\mathbb{P}$ that $\vdash_{\operatorname{Add}(\omega, 1)} "\left|\dot{\mathbb{P}}^{0}\right|<\delta$ ". Thus, by the results of $[10], V^{\mathbb{P}}=V^{\operatorname{Add}(\omega, 1) *\left(\dot{\mathbb{P}}^{1} \times \dot{\mathbb{P}}^{0}\right)} \vDash " \delta$ is $\lambda$ strongly compact" iff $V^{\operatorname{Add}(\omega, 1) * \dot{\mathbb{P}}^{1}} \vDash " \delta$ is $\lambda$ strongly compact". It is hence true that $V^{\operatorname{Add}(\omega, 1) * \dot{\mathbb{P}}^{1}} \vDash$ " $\delta$ is $\lambda$ strongly compact".

By its definition, $\operatorname{Add}(\omega, 1) * \dot{\mathbb{P}}^{1}$ is such that $|\operatorname{Add}(\omega, 1)|=\omega, \operatorname{Add}(\omega, 1)$ is nontrivial, and $\Vdash_{\operatorname{Add}(\omega, 1)}$ "ن্P 1 is $\aleph_{2}$-directed closed". In addition, by the same analysis as given in the preceding paragraph, if $\gamma \in A_{2}$, then $F(\gamma)<\delta$ (so in particular, since $A_{0} \cup A_{2}=\{\gamma \in A \mid \gamma<\delta\}$, we infer that $F(\gamma)<\delta$ for every $\gamma<\delta$ ). From this, the factorization in $V_{0}$ of $\mathbb{P}^{1}$ as $\mathbb{Q}^{0} \times \mathbb{Q}^{1}$, and the fact that both $\mathbb{Q}^{0}$ and $\mathbb{Q}^{1}$ are Easton products, it further follows that $\operatorname{Add}(\omega, 1) * \dot{\mathbb{P}}^{1}$ is mild with respect to $\delta$. Consequently, by Theorem $5 . V \vDash$ " $\delta$ is $\lambda$ strongly compact". Because level by level equivalence holds in $V$, $V \vDash$ "Either $\delta$ is $\lambda$ supercompact, or $\delta$ is a measurable limit of cardinals $\eta$ which are $\lambda$ supercompact".

If $V \vDash$ " $\delta$ is $\lambda$ supercompact", then by Lemma 2.2. $V^{\mathbb{P}} \vDash$ " $\delta$ is $\lambda$ supercompact". If $V \vDash$ " $\delta$ is a measurable limit of cardinals $\eta$ which are $\lambda$ supercompact", then let $\eta_{0}<\delta$ be least such that $V \vDash$ " $\eta_{0}$ is $\lambda$ supercompact". By its definition, there is no $\gamma \in A$ with $\eta_{0} \leq \gamma \leq \lambda^{+}$. Thus, we can write $A=A_{4} \cup A_{5}$, where $A_{4}=\left\{\gamma \in A \mid \gamma<\eta_{0}\right\}$ and $A_{5}=\left\{\gamma \in A \mid \gamma \geq \lambda^{++}\right\}$. If $\gamma \in A_{4}$, then $\gamma<\eta_{0}<\delta<\lambda$. Hence, by the same analysis as in the preceding two paragraphs, $F(\gamma)<\delta$. Also, by its definition, $\left|A_{4}\right| \leq \eta_{0}$. This means that if we define in $V_{0}$

$$
\mathbb{Q}^{2}=\prod_{\gamma \in A_{4}} \operatorname{Add}(\gamma, F(\gamma)) \quad \text { and } \quad \mathbb{Q}^{3}=\prod_{\gamma \in A_{5}} \operatorname{Add}(\gamma, F(\gamma)),
$$

then $V_{0} \vDash "\left|\mathbb{Q}^{2}\right|<\delta$ and $\mathbb{Q}^{3}$ is $\lambda^{++}$-directed closed". It consequently follows from the results of [10] that $V_{0}^{\mathbb{Q}^{2}} \vDash$ " $\delta$ is a measurable limit of cardinals $\eta$ which are $\lambda$ supercompact". Because GCH in $V_{0}$ yields $V_{0}^{\mathbb{Q}^{2}} \vDash " 2^{\gamma}=\gamma^{+}$for all cardinals $\gamma>\left|\mathbb{Q}^{2}\right| "$, we see that $\left.V_{0}^{\mathbb{Q}^{2}} \vDash \cdot 2^{[\lambda]}\right]^{<\delta}=2^{\lambda}=\lambda^{+}$". Again by $[\underline{8}$, Lemma 15.19], as in the proof of Lemma 2.2, we may now infer that forcing 
with $\mathbb{Q}^{3}$ over $V_{0}^{\mathbb{Q}^{2}}$ adds no new subsets of $2^{[\lambda]^{<\delta}}$. This means that $V_{0}^{\mathbb{Q}^{2} \times \mathbb{Q}^{3}}=$ $V^{\mathbb{P}} \vDash " \delta$ is a measurable limit of cardinals $\eta$ which are $\lambda$ supercompact". Consequently, we have that $V^{\mathbb{P}} \vDash$ "Either $\delta$ is $\lambda$ supercompact, or $\delta$ is a measurable limit of cardinals $\eta$ which are $\lambda$ supercompact", i.e., $V^{\mathbb{P}} \vDash$ "Either level by level equivalence holds at $\delta$, or $\delta$ is a witness to the Menas exception at $\lambda "$.

Cases 1-3 complete the proof of Lemma 2.3.

We note that the same analysis given in the first paragraph of Case 3 of Lemma 2.3 allows us to infer that if $V^{\mathbb{P}}=V^{\operatorname{Add}(\omega, 1) *\left(\dot{\mathbb{P}}^{1} \times \dot{\mathbb{P}}^{0}\right)} \vDash " \delta$ is $\lambda$ supercompact", then $V^{\operatorname{Add}(\omega, 1) * \dot{\mathbb{P}}^{1} \vDash} \vDash \delta$ is $\lambda$ supercompact" as well. We will make use of this fact in the proof of Lemma 2.4 .

LEMMA 2.4. $V^{\mathbb{P}} \vDash$ "K is the class of supercompact cardinals".

Proof. We adopt the notation of the preceding lemmas. Since $|\mathbb{P}|=\kappa$, by the results of $\left[10, V^{\mathbb{P}} \vDash\right.$ "The class of supercompact cardinals above $\kappa$ is the same as in $V^{\prime}$, i.e., $V^{\mathbb{P}} \vDash$ " $\mathcal{K} \backslash\{\kappa\}$ is the class of supercompact cardinals above $\kappa$ ". We also know that by Case 2 of Lemma 2.3, $V^{\mathbb{P}} \vDash$ " $\kappa$ is supercompact". Thus, to complete the proof of Lemma 2.4, it suffices to show that $V^{\mathbb{P}} \vDash$ "No cardinal $\delta<\kappa$ is supercompact"

To see that this is true, suppose to the contrary that $V^{\mathbb{P}} \vDash " \delta<\kappa$ is supercompact". Since $V \vDash$ " $\kappa$ is the least supercompact cardinal", let $\lambda>\delta$ be such that $V \vDash$ " $\delta$ is not $\lambda$ supercompact". We must have that $V^{\mathbb{P}} \vDash$ " $\delta$ is $\lambda$ supercompact". As we have just observed, the analysis given in Case 3 of Lemma 2.3 shows that $V^{\mathbb{P}}=V^{\operatorname{Add}(\omega, 1) *\left(\dot{\mathbb{P}}^{1} \times \dot{\mathbb{P}}^{0}\right)} \vDash " \delta$ is $\lambda$ supercompact" iff $V^{\operatorname{Add}(\omega, 1) * \mathbb{P}^{1}} \vDash " \delta$ is $\lambda$ supercompact". The factorization of $\operatorname{Add}(\omega, 1) * \dot{\mathbb{P}}^{1}$ given in the first sentence of the second paragraph of Case 3 in Lemma 2.3 together with Theorem 5 then show that $V \vDash$ " $\delta$ is $\lambda$ supercompact", a contradiction. This completes the proof of Lemma 2.4.

Since by its definition, $\mathbb{P}$ is $\kappa$-c.c., by [9, Exercise H2, p. 247], $A$ remains stationary in $V^{\mathbb{P}}$. Lemmas 2.1 2.4 and the intervening remarks therefore complete the proof of Theorem 4 .

As in [1] and 2, we conclude by asking what other types of Easton theorems are consistent with the level by level equivalence between strong compactness and supercompactness. In particular, is it possible to include regular cardinals in the domain of the Easton function $F$ which are above the least supercompact cardinal $\kappa$ ? This is precluded by the restrictions on $A$ given in Theorem 4. Is it possible for F's domain to be all regular cardinals, as in Easton's original result? Which cardinals may be included in F's range? Finally, are there any GCH patterns which are incompatible with the level by level equivalence between strong compactness and supercompactness? 


\section{References}

[1] A. Apter, An Easton theorem for level by level equivalence, Math. Logic Quart. 51 (2005), 247-253.

[2] A. Apter, More Easton theorems for level by level equivalence, Colloq. Math. 128 (2012), 69-86.

[3] A. Apter, On the non-extendibility of strongness and supercompactness through strong compactness, Fund. Math. 174 (2002), 87-96.

[4] A. Apter and J. Cummings, Identity crises and strong compactness II: Strong cardinals, Arch. Math. Logic 40 (2001), 25-38.

[5] A. Apter and S. Shelah, On the strong equality between supercompactness and strong compactness, Trans. Amer. Math. Soc. 349 (1997), 103-128.

[6] J. D. Hamkins, Gap forcing, Israel J. Math. 125 (2001), 237-252.

[7] J. D. Hamkins, Gap forcing: Generalizing the Lévy-Solovay theorem, Bull. Symbolic Logic 5 (1999), 264-272.

[8] T. Jech, Set Theory: The Third Millennium Edition, Revised and Expanded, Springer, Berlin, 2003.

[9] K. Kunen, Set Theory: An Introduction to Independence Proofs, Stud. Logic Found. Math. 102, North-Holland, Amsterdam, 1980.

[10] A. Lévy and R. Solovay, Measurable cardinals and the continuum hypothesis, Israel J. Math. 5 (1967), 234-248.

[11] T. Menas, Consistency results concerning supercompactness, Trans. Amer. Math. Soc. 223 (1976), 61-91.

[12] T. Menas, On strong compactness and supercompactness, Ann. Math. Logic 7 (1974), 327-359.

Arthur W. Apter

Department of Mathematics

Baruch College of CUNY

New York, NY, 10010, U.S.A.

and

The CUNY Graduate Center, Mathematics

365 Fifth Avenue

New York, NY, 10016, USA

E-mail: awapter@alum.mit.edu

http://faculty.baruch.cuny.edu/aapter 